\title{
Comments on Hadron Mass Dependence in Quark Models (*).
}

\author{
L. GoodmaN \\ Drexel University - Philadelphia, Pa.
}

(ricevuto il 9 Marzo 1970; manoscritto revisionato ricevuto il 24 Aprile 1970)

Summary. - A inodified harmonic-oscillator potential provides dynamics consistent with known empirical information in the nonrelativistic quark model. The composite mass depends linearly on the constituent mass for deviations about the constituent mass in the Dirac quark model.

In the nonrelativistic quark model the dynamical mechanism underlying quark bound states is described by a hierarchy of forces ( $\left.{ }^{1}\right)$. The Hamiltonian for the most dominant of these forces, the so-called "superstrong" ones, is

$$
\begin{gathered}
H=\frac{1}{2} \sum_{i=1}^{n} p_{i}^{2}+n M+I^{\left(n^{\prime}, n-n^{\prime}\right)}, \\
V^{\left(n^{\prime}, n-n^{\prime}\right)} \equiv V^{\left(n^{\prime}, n-n^{\prime}\right)}\left(x_{1}, \ldots, x_{n} ; \sigma_{1}, \ldots, \sigma_{n}\right),
\end{gathered}
$$

in which the $\sigma_{i}$ are Pauli matrices for each particle, $p_{i} \equiv-i \nabla_{i}$ and $M$ is the mass of each constituent particle. We have set $\hbar=c=1$.

For a potential homogeneous under scale transformations,

$$
V^{\left(n^{\prime}, n-n^{\prime}\right)}\left(\lambda x_{i}, \ldots, \lambda x_{n} ; \sigma_{1}, \ldots, \sigma_{n}\right)=\lambda^{\gamma} V^{\left(n^{\prime}, n-n^{\prime}\right)}\left(x_{1}, \ldots, x_{n} ; \sigma_{i}, \ldots, \sigma_{n}\right)
$$

for all real $\lambda>0$, we can establish a mass relation. This is accomplished by introducing the dimensionless variables $\varepsilon \equiv(m-3 M)^{\gamma /(2+\gamma)}$ and $\boldsymbol{\xi}_{i} \equiv \boldsymbol{x}_{i}(2 M)^{1 /(2+\gamma)}$

(*) Work supported by a National Science Foundation Grant.

(1) J. J. J. KoKKedeE: The Quark Model (New York and Amsterdam, 1969). p. 27. 
in the equation $H \psi=m \psi$ from which it follows that

$$
\varepsilon=\varepsilon_{1}^{(2)}+\varepsilon_{2}^{(-\gamma)},
$$

where

and

$$
\varepsilon_{1}^{(2)}=\frac{\left(\psi\left(\boldsymbol{\xi}_{1}, \ldots, \boldsymbol{\xi}_{n}\right),-\sum_{i=1}^{n} \nabla_{\boldsymbol{\xi}_{i}}^{2} \psi\left(\boldsymbol{\xi}_{1}, \ldots, \boldsymbol{\xi}_{n}\right)\right)}{\left(\psi\left(\boldsymbol{\xi}_{1}, \ldots, \boldsymbol{\xi}_{n}\right), \psi\left(\boldsymbol{\xi}_{1}, \ldots, \boldsymbol{\xi}_{n}\right)\right)}
$$

$$
\varepsilon_{2}^{(-\gamma)} \equiv \frac{\left(\psi\left(\xi_{1}, \ldots, \xi_{n}\right) V^{\left(n^{\prime}, n-n^{\prime}\right)} \psi\left(\boldsymbol{\xi}_{1}, \ldots, \boldsymbol{\xi}_{n}\right)\right)}{\left(\psi\left(\xi_{1}, \ldots, \xi_{n}\right), \psi\left(\boldsymbol{\xi}_{1}, \ldots, \boldsymbol{\xi}_{n}\right)\right)}
$$

The superscript on the energy terms refer to the degree of homogeneity of the functional under scale transformations. Using the pseudovirial theorem $\left(^{2}\right)$ on (2) it follows that

$$
\varepsilon_{1}^{(2)}=\frac{\gamma}{2} \varepsilon_{2}^{(-\gamma)}
$$

and

$$
\varepsilon=\frac{2+\gamma}{\gamma} \varepsilon_{1}^{(2)}
$$

Since $\varepsilon_{1}^{(2)} \geqslant 0$, we find that $\varepsilon<0$ for $-2<\gamma<0$ and $\varepsilon \geqslant 0$ otherwise.

From the definition of $\varepsilon, m=\varepsilon(2 M)^{-\gamma /(2+\gamma)}+3 M$ and the only integer value of $\gamma$ which produces binding (that is $m<M$ ) is $\gamma=-1$, in which case

$$
m=(2 \varepsilon+3) M
$$

Since it is known that $M \geqslant 10 \mathrm{GeV}$, eq. (5) implies that the system is in the relativistic domain. Thus, $\gamma=-1$ is also excluded from consideration.

Several authors ( $\left.{ }^{3.4}\right)$ have suggested a harmonic-oseillator model for baryons. Such a model is not compatible with $m / M \leqslant 1 / 10$. A more realistic model is one in which there is a minimum range of interaction. A potential which accomplishes this is

$$
V=\frac{1}{2} K \sum_{i<j}\left(\left|\boldsymbol{x}_{i}-\boldsymbol{x}_{j}\right|-a\right)^{2}-\frac{1}{2} K a^{2}=\frac{1}{2} K \sum_{i<j}\left(\left|\boldsymbol{x}_{i}-\boldsymbol{x}_{j}\right|^{2}-2 a\left|\boldsymbol{x}_{i}-\boldsymbol{x}_{j}\right|\right)
$$

In the Appendix it is shown that if the potential is composed of two terms, one of homogeneity with respect to scale transformations of order 2 , the other

(2) G. Rosen: Journ. Math. Phys.. 7, 2066 (1966).

(3) D. Faiman and A. W. Hendry: Phys. Rev.. 173, 1720 (1968).

(4) T. C. Navarko. M. Moshinsky and W. W. Y.ri: Revista Mexicana de Fisica. $17.4,241$ (1968). 
of order $\gamma$, for binding to occur consistent with $m / M \leqslant 1 / 10$ the potential must be similar to the above one. For the type of potential given by (6) one expects $n M=\frac{1}{2} K a^{2}$ consistent with <kinetic energy' $<m$.

For the case $n=2$ (mesons) it is straightforward to establish the mass formula

$$
m=C M^{-\frac{1}{2}}+2 M-\frac{1}{2} K a^{2},
$$

where $C \equiv\left(l+2 k+\frac{3}{2}\right) K^{\frac{1}{2}}, l$ is the orbital angular-momentum quantum number, and the parameter $k$ is associated with the number of nodes of the wave function. For the $J^{P}=1^{-}$mesons of mass $m=760 \mathrm{MeV}$ the requirement $\langle$ kinetic energy $\rangle=\frac{1}{2} C M^{-\frac{1}{2}}<m$ along with assumptions $M \approx 10 \mathrm{GeV}$ and and $2 M \approx \frac{1}{2} K a^{2}$ yields the inequality $C<1.4 \cdot 10^{5}(\mathrm{MeV})^{\frac{3}{2}}$. For a meson in the ground state our model predicts $a>2.1 \cdot 10^{-3}(\mathrm{MeV})^{-1}$.

The dynamics of this particular model deserves further study. It has been shown $\left(^{3}\right)$ that a paraquark harmonic-oscillator shell model can explain the band structure of the known $\mathcal{N}^{3 *}$ resonances and $\mathcal{N} \pi$ and $\Delta \pi$ partial decay widths.

Let us now consider the Dirac quark-model Hamiltonian for a system of $n^{\prime}$ quarks and $n-n^{\prime}$ antiquarks,

$$
\left\{\begin{array}{l}
H=\sum_{i=1}^{n}\left(\boldsymbol{\alpha}_{i} \cdot \boldsymbol{p}_{\imath}+\beta_{i} M_{i}\right)+V^{\left(\boldsymbol{n}^{\prime}, n-n^{\prime}\right)}, \\
V^{\gamma\left(n^{\prime}, n-n^{\prime}\right)}=V^{r}\left(\boldsymbol{x}_{1}, \ldots, \boldsymbol{x}_{n} ; \boldsymbol{\alpha}_{1}, \ldots, \boldsymbol{\alpha}_{n}, \beta_{1}, \ldots, \beta_{n}\right),
\end{array}\right.
$$

in which the $\boldsymbol{\alpha}$ 's and $\beta$ 's are Dirac matrices, $\boldsymbol{p}_{i}=-i \boldsymbol{\nabla}_{\imath}$, and $\boldsymbol{M}_{\boldsymbol{i}}$ is the mass of the $i$-th particle. For a potential homogeneous under scale transformations, we have

$$
\begin{aligned}
V^{\left(n^{\prime}, n-n^{\prime}\right)}\left(\lambda x_{1}, \ldots, \lambda x_{n} ; \boldsymbol{\alpha}_{1}, \ldots, \boldsymbol{\alpha}_{n}, \beta_{1}, \ldots, \beta_{n}\right)= & \\
& =\lambda^{\gamma} V^{\left(n^{\prime}, n-n^{\prime}\right)}\left(\boldsymbol{x}_{1}, \ldots, \boldsymbol{x}_{n} ; \boldsymbol{\alpha}_{1}, \ldots, \boldsymbol{\alpha}_{n}, \beta, \ldots, \beta_{n}\right) .
\end{aligned}
$$

From the pseudovirial theorem $\left({ }^{2}\right)$ it follows that

$$
m=\frac{1+\gamma}{\gamma} E_{1}^{(1)}+E_{2}^{(0)}
$$

where

$$
m=\frac{(\psi, H \psi)}{(\psi, \psi)}
$$

$$
E_{1}^{(1)} \equiv \frac{\left(\psi, \sum_{i=1}^{n} \boldsymbol{\alpha}_{i} \cdot \boldsymbol{p}_{i} \psi\right)}{(\psi, \psi)}, \quad E_{\mathbf{2}}^{(0)} \equiv \sum_{i=1}^{n} M_{i} \frac{(\psi, B, \psi)}{(\psi, \psi)}, \quad E_{\mathbf{3}}^{(-\gamma)}=\frac{\left(\psi, V^{\left(n, n-n^{\prime}\right)} \psi\right)}{(\psi, \psi)} .
$$


We have employed similar notation to that previously used. If the system is in the ground state, then the pseudovirial theorem may be extended ( $\left.{ }^{5}\right)$ to establish the inequality

$$
\gamma(\gamma+1) E_{3}^{(-\gamma)} \geqslant 0
$$

Consider the following cases:

$$
\begin{array}{rlll}
\text { I) } \gamma<-1, & E_{3}^{(-\gamma)} \geqslant 0, & E_{1}^{(1)}<0, & E_{2}^{(0)} \geqslant 0, \\
\text { II) } 0>\gamma>-1, & E_{3}^{(-\gamma)} \leqslant 0, & E_{1}^{(1)} \geqslant 0, & E_{2}^{(0)} \geqslant 0, \\
\text { III) } \gamma>0, & E_{3}^{(-\gamma)} \geqslant 0, & E_{1}^{(1)} \geqslant 0 . &
\end{array}
$$

We note that

$$
\frac{\partial m}{\partial M_{i}}=\frac{\left(\psi,\left(\partial H / \partial M_{i}\right) \psi\right)}{(\psi, \psi)}=\frac{\left(\psi, B_{i} \psi\right)}{(\psi, \psi)} \equiv C_{i}
$$

Differentiating (9), and using the fact that $E_{2}^{(\mathbf{0})}==\sum_{j=1}^{n} M_{\imath} C_{\imath}$ leads to

$$
\frac{1+\gamma}{\gamma} \frac{\partial E_{1}^{(1)}}{\partial M_{i}}=-\sum_{i=1}^{n} M_{j} \frac{\hat{\partial} C_{j}}{\hat{c} M_{i}}
$$

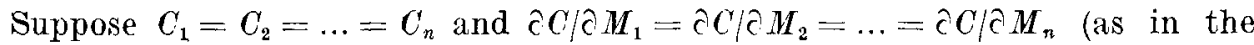
case of mutually alligned spins).

Then, (12) reduces to

$$
\frac{1+\gamma}{\gamma} \frac{\partial E_{1}^{(1)}}{\partial M_{i}}=-M \frac{\partial G}{\partial M_{i}}=M \frac{\partial^{2} m}{\partial M_{i}^{2}},
$$

in which $M$ is defined by

$$
M \equiv \sum_{i=1}^{n} M_{i}
$$

From Schwartz's inequality $\left({ }^{6}\right)$ we establish the inequality

$$
\frac{\partial m}{\partial M_{i}}=\frac{(\psi, B, \psi)}{(\psi, \psi)} \leqslant \frac{\left(\psi, B_{i}^{2} \psi\right)(\psi, \psi)^{\frac{1}{2}}}{(\psi, \psi)}=1 .
$$

$\left(^{5}\right)$ For a variation around the ground state $\left(\mathrm{d}^{2} / \mathbf{d} \lambda^{2}\right) m\left[\left.\psi(\hat{\lambda} \boldsymbol{x} i)^{7}\right|_{\lambda-1} \geqslant 0\right.$ since $(\psi \cdot H \psi) /(\psi, \psi) \geqslant E_{0}$.

${ }^{6}{ }^{6}$ G. Rosen: Mass dependence in the Dirac quark model (preprint). 
If $\hat{c}^{2} \mathrm{~m} / \mathrm{c} \boldsymbol{M}_{i}<\infty$ it follows from (13) that for $M \sim 0, E_{1}^{(1)} \sim A M^{n}, n \geqslant 2, A$ a constant independent of $\boldsymbol{M}_{i}$, and hence that $\quad C=E_{2}^{(\mathbf{0})} / M=\hat{c} m / \partial M_{i} \sim B M^{n-1}$ with $B=-((1+\gamma) / \gamma)(n /(n-1)) A$. Substituting both of these limiting valnes for $E_{1}^{(1)}$ and $E_{2}^{(0)}$ into (9) implies that, as $M \rightarrow 0, m \rightarrow 0$. This cannot be immediately deduced from (8). Case III) $\left(\gamma>0 E_{2}^{(0)} \geqslant 0\right)$ can be eliminated, since near $M=0$ we have $A>0$ which implies that $\beta>0$ and hence that $\partial m / \partial M_{1}<0$. Thus we conclude $m(\mathbf{0})=0^{-}$.

For eases I) and II) note that c $m / \partial M_{i}>0$ for $M_{i}>0$. From (13) we see that large inflections are damped out by the $M^{-1}$ factor. This suggests

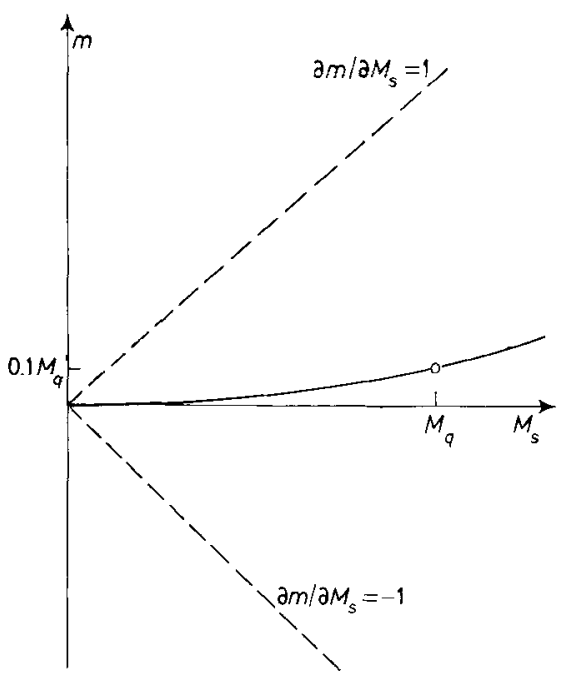

Fig. 1. - A plot of composite mass $(m)$ rs. constituent mass $\left(M_{l}\right)$ in units of the quark mass, $M_{q} \approx 10 \mathrm{GeV}$. the mass relation shown in Fig. 1. Such a relationship lends credence to the use of (11) to find the mass dependence of the composite system about the constituent mass point $M_{i}=M_{q}$. An attempt has been made to employ the quasi-independent model to obtain such an approximation of $(11)\left({ }^{6}\right)$.

净* * *

I would like to thank Prof. G. Rosex for a number of most helpiul disenssions.

APPEXDIX

Consider the nonrelativistic Hamiltonian

$$
H=\sum_{i=1}^{n} \frac{\boldsymbol{p}_{i}^{2}}{2} \bar{M}^{2}+n M+\frac{1}{2} K \sum_{i<j}\left|\boldsymbol{x}_{\imath}-\boldsymbol{x}_{j}\right|^{2}+\boldsymbol{V}^{\prime},
$$

in which $I^{\prime \prime}$ is defined by

$$
\Gamma^{\prime \prime} \equiv \Gamma^{\prime}\left(x_{1}, \ldots, x_{n} ; \sigma_{1}, \ldots, \sigma_{n}\right)
$$

and

$$
V^{\prime}\left(\lambda \boldsymbol{x}_{1}, \ldots, \lambda \boldsymbol{x}_{n} ; \boldsymbol{\sigma}_{1}, \ldots, \boldsymbol{\sigma}_{n}\right)=\lambda^{\gamma} V^{\prime}\left(\boldsymbol{x}_{1}, \ldots, \boldsymbol{x}_{n} ; \boldsymbol{\sigma}_{1}, \ldots, \boldsymbol{\sigma}_{n}\right)
$$


We establish the mass relation

$$
m=E_{1}^{(2)}+E_{2}^{(0)}+E_{3}^{(-2)}+E_{4}^{(-\gamma)}
$$

$m=$ mass of the composite system, $E_{1}^{(2)}, E_{2}^{(0)}, E_{3}^{(-2)}, E_{4}^{(-y)}$ defined by

$$
\begin{aligned}
& E_{1}^{(2)}=\frac{\left(\psi, \sum_{i=1}^{n}\left(\boldsymbol{p}_{i}^{2} / 2 M\right) \psi\right)}{(\psi, \psi)}, \quad E_{2}^{(0)}=n M \\
& E_{\mathbf{3}}^{(-\mathbf{2})}=\frac{\left(\psi, \frac{1}{2} K \sum_{i<\boldsymbol{j}}\left|\boldsymbol{x}_{\boldsymbol{i}}-\boldsymbol{x}_{\boldsymbol{j}}\right|^{2} \psi\right)}{(\psi, \psi)}, \quad E_{\mathbf{4}}^{\left(-\gamma^{\prime}\right)}=\frac{\left(\psi, V^{\prime} \psi\right)}{(\psi, \psi)} .
\end{aligned}
$$

From the pseudovirial theorem $\left({ }^{2}\right)$ it follows from (A.2) that

$$
2 E_{1}^{(2)}-2 E_{3}^{(-2)}-\gamma E_{4}^{(-1)}=0
$$

Assuming the system to be in the ground state $\left(^{5}\right)$ a second variation of (A.2) is used to yield the inequality

$$
2 E_{1}^{(2)}+6 E_{3}^{(-2)}+\gamma(\gamma+1) E_{4}^{(-\gamma)} \geqslant 0 \text {. }
$$

Combining (A.2) and (A.3) with (A.4) we find

$$
(\gamma+2) E_{1}^{(2)} \geqslant(\gamma-2) E_{3}^{(-2)}
$$

For $\gamma<2$ we have

$$
E_{3}^{(-2)} \geqslant \frac{\gamma+2}{\gamma-2} E_{1}^{(2)}
$$

and for $\gamma>2$ we have

$$
E_{3}^{(-2)} \leqslant \frac{\gamma+2}{\gamma-2} E_{1}^{(2)}
$$

Substituting (A.3) into (A.2) produces

$$
m=\frac{\gamma+2}{\gamma} E_{\mathrm{L}}^{(2)}+\frac{\gamma-2}{\gamma} E_{3}^{(-2)}+n M .
$$

From (A.2) we note that no binding can occur for $\gamma \geqslant 2$ and $\gamma<-2$ since

$$
\frac{\gamma+2}{2} E_{2}^{(2)} \geqslant 0 \quad \text { and } \quad \frac{\gamma-2}{2} E_{3}^{(-2)} \geqslant 0
$$


Consider the following three eases.

Case I) $\gamma=-1\left(^{7}\right)$. Equation (A.7) reduces to

$$
m=-E_{1}^{(2)}+3 E_{3}^{(-2)}+n M
$$

and from (A.6a) it follows that $E_{3}^{(-2)} \geqslant-\frac{1}{3} E_{1}^{(2)}$ and hence $m \geqslant n M$.

Case II) $\gamma=-2$. Equation (A.7) reduces to

$$
m=2 E_{3}^{(-2)}+n M
$$

and since $E_{3}^{(-2)} \geqslant 0$ then again we have $m \geqslant n M$.

Case III) $\gamma=1$. From (A.6a), (A.2) and (A.3) we find

$$
m=2 E_{1}^{(2)}-E_{3}^{(-2)}+n M=\frac{3}{2} E_{4}^{-1}+2 E_{3}^{(-2)}+n M .
$$

Substituting (A.3) into (A.9) yields

$$
\text { (A.11a) }
$$

$$
E_{3}^{(-2)}+3 E_{1}^{(2)} \geqslant 0
$$

or

$$
E_{4}^{(-1)}+\frac{8}{3} E_{3}^{(-2)} \geqslant 0
$$

We can have $m<n M$ if the inequality

$$
-\frac{8}{3} E_{3}^{(-2)} \leqslant E_{4}^{(-1)} \leqslant-\frac{4}{3} E_{3}^{(-2)}
$$

holds.

We note that $E_{4}^{(-1)}$ cannot be found by a perturbation calculation.

(7) The case $\gamma=-1$ is considered in ref. $\left({ }^{6}\right)$.

\section{RIASSUNTO}

Un potenziale modificato dell'oscillatore armonico fornisce una dinamica consistente con note informazioni empiriche sul modello a quark non relativistico. La massa composta dipende linearmente dalla massa costituente per deviazioni intorno alla massa costituente nel modello a quark di Dirac.

(*) Traduzione a cura della Reduzione.

Замечания о зависимости от адронных масс в моделях кварков.

Резюме $\left({ }^{*}\right)$. - Модифицированный потенциал гармонического осциллятора обеспечивает динамику, согласуюшуюся с известной эмпирической информацией в нерелятивистской модели кварков. В дираковской модели кварков составная масса линейно зависит от массы составляющих частиц для отклонений, порядка массы составляющих частиц.

(•) ПТереведено редакцией. 